\title{
Clinical Trials of DNA and Recombinant Adenovector (rAd) Vaccines for HIV Barney S Graham*‡
}

\author{
Address: Vaccine Research Center, NIAID, NIH, Bethesda, MD \\ Email: Barney S Graham* - bgraham@nih.gov \\ * Corresponding author ‡Presenting author
}

\author{
from 2005 International Meeting of The Institute of Human Virology \\ Baltimore, USA, 29 August - 2 September 2005 \\ Published: 8 December 2005 \\ Retrovirology 2005, 2(SuppI I):S6I doi:I0.II86/1742-4690-2-SI-S6I
}

\section{Background}

Gene-based vaccine delivery is an important strategy for induction of $\mathrm{T}$ cell responses that may be critical for a successful AIDS vaccine. Despite promising results in animal models, evidence of immune responses to DNA and rAd vaccines in humans has been limited.

\section{Materials and methods}

Three Phase I studies have evaluated a series of DNA and rAd vaccine candidates expressing constructs encoding clades A, B, and C Envelope and clade B Gag and Pol with or without Nef, as fusion proteins or individually.

\section{Results}

T cell and antibody responses are detected by IFN- $\alpha$ ELISpot and FACS detection of intracellular IL-2 or IFN- $\alpha$ in the large majority of vaccinees. Env peptide pools elicit the strongest response, but the 6-plasmid and rAd product also induced robust responses to Gag, Pol, and Nef. Both $\mathrm{T}$ cell and humoral responses were dose dependent. The $\mathrm{T}$ cell responses induced by DNA are detectable for at least 52 weeks, and the pattern of cytokine expression evolves over time with fewer IFN- $\gamma$ and more IL- 2 producing T cells at one year.

\section{Conclusion}

DNA and rAd5 vaccine candidates are well tolerated and induce broad, durable immune responses. The combination will be tested in Phase II trials beginning 4Q2005. 